\title{
Reform and Implementation of Experiment on Adsorption of Active Carbon to Phenol
}

\author{
Shuqin Wang ${ }^{*}$, and Jinjin Wu \\ Department of Environmental Science and Engineering, \\ North China Electric Power University \\ Baoding, 071003, China \\ *E-mail: wsqhg@163.com
}

\begin{abstract}
According to the "Water Pollution Control Engineering" course, the activated carbon adsorption experiment was comprehensive reformed; the details of the experiment were optimized; the students' practical ability was improved; the students were guided to explore experiments, and the theoretical knowledge was combined with practical problems to stimulate students to carry out scientific experiments. The results show that the comprehensive reform of phenol adsorption on activated charcoal facilitates the improvement of students' enthusiasm for learning, and it also contributes to the improvement of students' overall quality.
\end{abstract}

Keywords: activated carbon; comprehensive experiment; adsorption performance; teaching reform;

\section{INTRODUCTION}

The Research Teaching Model is the key to the reform of university teaching methods in recent years [1]; and it is one of the successful models for the cultivation of innovative talents. Water Pollution Control Engineering is the core course and school-level exquisite course of Environmental Engineering of North China Electric Power University. This course focuses on the basic concepts, basic theory, treatment process, process design, and other related knowledge related to wastewater treatment. At present, there have been many advances in the research on the reform of the curriculum system [2]. Related explorations have also been carried out, but there are fewer reforms in the practice [3]. In order to cultivate students' practical hands-on and innovative abilities, existing experimental projects have undergone design reforms [3]. Guide students to actively participate in teaching, develop students' abilities to use professional knowledge to analyze problems and solve problems, and develop their comprehensive abilities such as inquiry research and teamwork. This comprehensive experiment - the activated carbon adsorption and removal of COD experiment has also been reformed, further cultivating their ability to analyze problems and solve problems, and eventually formed the awareness of autonomous learning and improved the ability to apply knowledge. However, the effect of COD removal by activated carbon is not satisfactory. It is also necessary to further optimize the experimental conditions. For this reason, the following two aspects will be reformed.

\section{REFORM OF DETERMINATION METHOD OF ACTIVATED CARBON ADSORPTION PROPERTY}

\section{A. Determination of Experimental Data Processing Model}

The activated carbon batch adsorption experiments in water pollution control engineering experiment textbooks require the addition of $0 \mathrm{mg}, 100 \mathrm{mg}, 500 \mathrm{mg}, 1000 \mathrm{mg}$, $1500 \mathrm{mg}$, and $2000 \mathrm{mg}$ of powdered activated carbon to determine the COD before and after adsorption of the wastewater. Calculate the amount of adsorption $q$ according to Ferrandlich's empirical formula (1):

$$
\mathrm{q}=\mathrm{K} \cdot \mathrm{C}^{1 / \mathrm{n}} \text {. }
$$

q---- Activated carbon adsorption, g/g

C----Adsorbed substance equilibrium concentration, $\mathrm{g} / \mathrm{L}$

K,n---- Constants

In recent years, experimental results have shown that students have large titration errors. After the data is plotted, they cannot obtain a beautiful straight line. The calculated slopes and intercepts are different and there is no law at all. For this reason students were asked to use the Langmuir isotherm simultaneously and plotted $\mathrm{C} / \mathrm{q}$ against $\mathrm{C}$. The slope of the straight line was $\mathrm{K}_{1} / \mathrm{K}$ and the intercept was $1 / K$. The adsorption isotherms obtained from the two determination methods and the two treatment models were plotted and compared. As shown in Figure 1.

It can be seen that the fitting of the two equations is relatively high, and the linear correlation coefficient $\mathrm{R}^{2}$ is greater than 0.96 , but the calculation of the Langumir equation is relatively simple. The potassium permanganate method is similar to the spectrophotometric method in terms of fitting, and is in an acceptable range. Therefore, spectrophotometric determination of phenol removal rate is simple and feasible. This method was used to conduct experiments. 


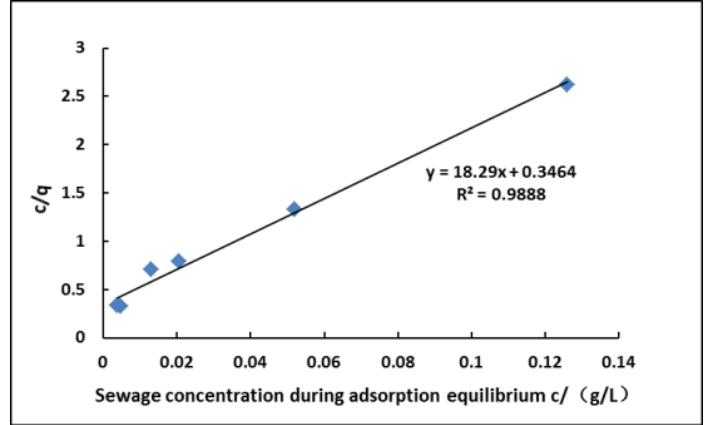

(A)

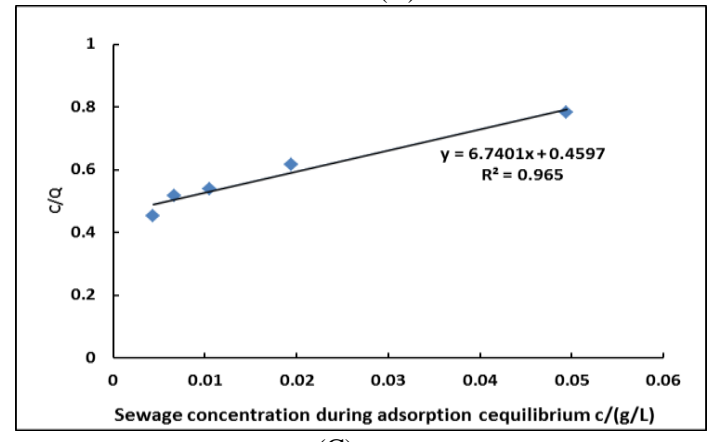

(C)

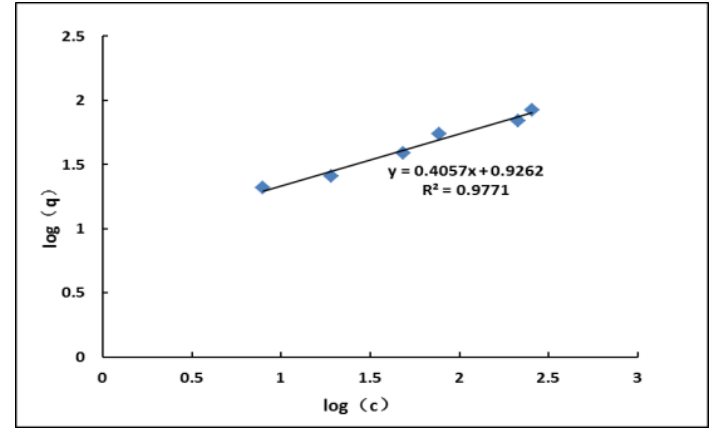

(B)

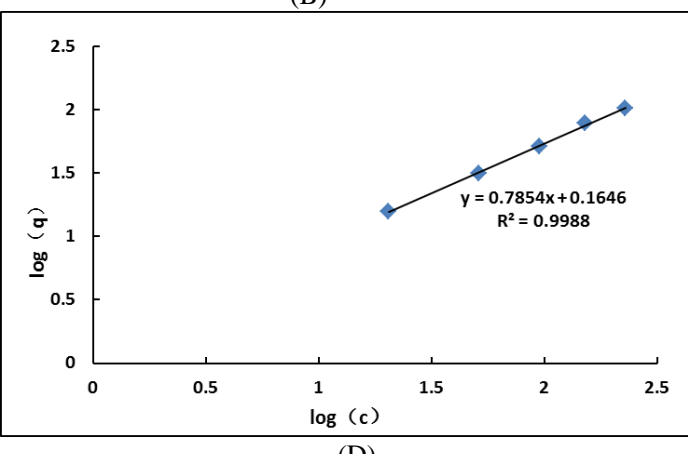

(D)

Fig. 1 Powdered activated carbon adsorption isotherms with different models which are Langmuir (Spectrophotometry) (A), Freundlich (Spectrophotometry) (B), Langmuir (potassium permanganate) (C) and Freundlich (potassium permanganate) (D)

\section{B. Selection of Test Methods}

The potassium dichromate method has the advantages of complete oxidation, accurate measurement, and good reproducibility; the disadvantage is that the measurement takes 2 hours; the amount of reagents is large, and mercury salts cause secondary pollution. The oxidation rate of potassium permanganate is low, but it is simple and can be used when the content of organic matter in water sample is relatively low. Potassium permanganate method for the determination of water samples ranged from 0.5 to 10 $\mathrm{mg} / \mathrm{L}$. The determination of COD by potassium permanganate can, to some extent, explain the state of organic contamination of water bodies. In the water pollution control engineering experiment textbook [4], the potassium permanganate method for the determination of COD in water is an experiment that was set up separately. It was fused to the activated carbon for the determination of COD adsorption performance, but it required self-contained wastewater containing organics and phenol was targeted. The spectrophotometric determination of phenol compared with potassium permanganate method requires simple equipment, simple and fast operation, high sensitivity and wide application. Therefore, spectrophotometry was selected for experiment.

\section{REFORM OF ADSORPTION PERFORMANCE OF DIFFERENT ACTIVATED CARBONS}

In order to reflect the comprehensiveness of the experiment for the determination of the adsorption performance of activated carbon, students are required to study the influence of the amount of activated carbon and the particle size of activated carbon on the adsorption performance from different aspects. However, there were only two different particle size comparisons in previous experiments, and there was no instrument to measure particle size. Therefore, the experiment was further reformed, and the adsorption of phenol on different activated carbons was supplied, and a particle size analyzer was purchased to measure the accurate particle size data. Then the experimental data are analyzed and sorted out to deepen the understanding of the adsorption process mechanism and improve students' ability to analyze problems.

In the following experiments, phenol was used as the simulated wastewater, and the absorbance was measured by a spectrophotometer. The maximum absorption wavelength was $510 \mathrm{~nm}$. 4-aminoantipyrine and potassium ferricyanide were used as color reagents, followed by data processing and analysis.

\section{A. Relationship Between Particle Size of Activated Carbon and Adsorption Performance of Activated Carbon}

Take 5 equal weights of large and small particles of activated carbon. Each grinding time is different (The initial sewage concentration of large granular activated carbon is $0.03 \mathrm{~g} / \mathrm{L}$ and The initial sewage concentration of small granular activated carbon is $0.1 \mathrm{~g} / \mathrm{L}$ ). The particle size of various particles was tested by automatic wet laser particle size analyzer (NKTG100-B). Compare the performance of activated carbon of different materials. The results are shown in Figure 2 and Table 1: 

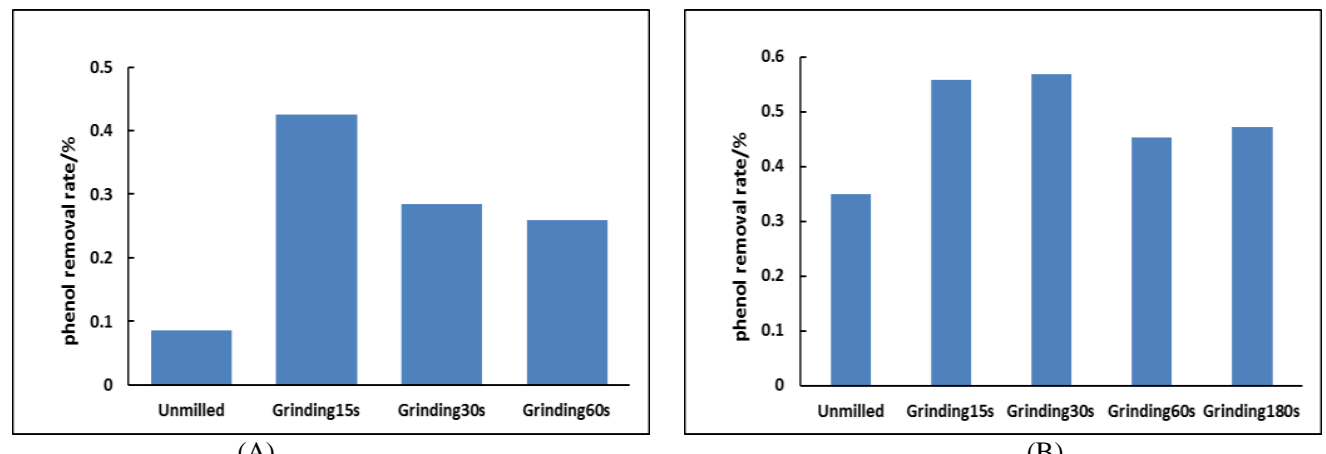

Fig.2 Effect of particle size of activated carbon on adsorption of phenol large granular activated carbon (A) and small granular activated carbon(B)

TABLE.1 GRANULARITY OF ACTIVATED CARBON GRINDING AT DIFFERENT TIMES

\begin{tabular}{cccc}
\hline Activated carbon & D10/ $\boldsymbol{\mu m}$ & $\mathbf{D 5 0} / \boldsymbol{\mu m}$ & $\mathbf{D 9 0} / \boldsymbol{\mu m}$ \\
\hline Small Grinding15s & 44.73 & 224.03 & 452.24 \\
Small Grinding 30s & 19.22 & 158.76 & 329.34 \\
Small Grinding 60s & 7.32 & 80.36 & 193.98 \\
Small Grinding 180s & 2.44 & 10.35 & 29.26 \\
Large Grinding 90s & 2.84 & 27.71 & 152.11 \\
Large Grinding 180s & 2.24 & 17.82 & 122.21 \\
Large Grinding 300s & 2.11 & 15.35 & 110.81 \\
powder & 8.51 & 26.24 & 70.27
\end{tabular}

From Figure 2 and Table.1, we know that the size of activated carbon will affect its adsorption performance, but not the finer the abrasive is, the better the adsorption performance is. After a long time of grinding of the activated carbon, the adsorption performance has been reduced. In the adsorption process, it is the microporous structure that really determines the adsorption capacity of activated carbon. All specific surfaces of activated carbon are almost composed of micropores. The macropores and transition pores only act as adsorption channels, but their presence and distribution affect the adsorption and desorption rates to a considerable extent [5].

\section{B. Comparison of Adsorption Performance of Different Types of Activated Carbon}

Different activated carbons are compared and the most suitable activated carbon is selected. Take $200 \mathrm{ml}$ of phenol solution whose concentration of $0.1 \mathrm{~g} / \mathrm{L}$ (Add $1 \mathrm{~g}$ of activated carbon, shock $1 \mathrm{~h}$ ). Phenol removal rate of coal large particles (Activated carbon) are $25 \%$. Wood particles are $93 \%$ and wood powders are $98 \%$.

According to the results, the adsorption effect of activated carbon with different materials on phenol is different, and the adsorption effect of wood activated carbon on phenol is the best [6], and the removal rate reaches over $90 \%$. The adsorption efficiency of phenol on coal-based activated carbon and wood-based activated carbon varies greatly. In some applications, the surface chemistry of activated carbon has a more important role than pore structure. It can be seen that wood activated carbon is an ideal material for adsorbing phenol.

Students can determine the particle size of activated carbon and the adsorption properties of different activated carbons in a comprehensive experiment. After these experiments, students can clearly understand the various factors affecting activated carbon adsorption, deepen the understanding of the adsorption process mechanism, and improve their ability to learn autonomously.

\section{CONCLUSIONS}

This teaching reform not only improves students' enthusiasm and creativity, but also helps students deepen their understanding and application of the basic theory of water pollution control. Through the comprehensive reform of traditional activated carbon experiments, it not only helps students to master the characteristics of adsorbents, the influence of various factors on the adsorption performance, but also cultivates students' ability to analyze, observe, and solve problems. Through the comparative analysis of experimental data, the theory and practice are more closely linked, improve their practical ability, and lay a foundation for improving students' innovation ability and engineering awareness.

\section{ACKNOWLEDGEMENT}

This work was financially supported by North China Electric Power University Fund (No. 130017013) and Hebei Province Fund (No. 13001805).

\section{REFERENCES}

[1] An-jun Chen. Research and Practice Based on Project-Research Teaching [J].Model Heilongjiang education, 2011(11):15-16.

[2] Wen-jie Zhang, Qing-lin Jie,Yue Jin. "Water Pollution Control Engineering" Course Assessment Method Reform and Practice [J]. Time Education, 2016, (1):18-24

[3] Shu-qin Wang, Ming-zhu Liu, Ke-xin Wang. Application of 
coagulation designing Experiment in Research - oriented teaching and learning [J]. Experimental Technology and Management, 2015, 3 (11) 31-34

[4] Ze-tang Chen. Water Pollution Control Engineering Experiment [M]. Chemical Industry Press, 2004

[5] Ding-ming Wang, Guo-ping Cao, Yun-fei Ja,Peng-cheng Liu. Application of Activated Carbon Adsorption Technology in Water Treatment [J]. Northern Environment, 2011, 23(11): 190-191.

[6] Rong Lu, Juan Tian, He Yingying, Zhao Junhuan, Chen Yanbin. Experimental Study of Absorption of Phenol and Methylene Blue with Different Modified Activated Carbons [J]. Environmental Protection Science , 2015, 44 (6):50-53. 